\title{
Abschließende Reflexionsfragen
}

\section{Timo Burger und Nicole Miceli}

Zur Vertiefung der im Kapitel Ausblicke dargestellten Themen, bieten sich noch folgende Reflexionsfragen für Lehramtsstudierende an, die sich möglichen Herausforderungen der empirischen Forschung im Kontext der Lehrer*innenbildung stellen möchten:

1. Reflektieren Sie, inwiefern die hier gezeigten Forschungsverläufe für Ihren eigenen Forschungsprozess relevant sein könnten.

2. Überlegen Sie sich, wie Sie aus einem Erkenntnisinteresse eine Fragestellung für Qualifikationsarbeiten und/oder kleinere Forschungsprojekte generieren könnten.

3. Wie könnten Sie gemachte Praxiserfahrungen (z.B. durch Praktika) im Rahmen einer Forschungsarbeit im universitären Kontext wissenschaftlich bearbeiten?

4. Reflektieren Sie, ob die eigene Forschungspraxis in der Lehrer*innenausbildung zu einem wissenschaftlich-reflexiven Habitus beitragen könnte. Welche Problematiken könnten in dieser Aufgabe liegen? 